\title{
Could symptoms and risk factors diagnose COPD? Development of a Diagnosis Score for COPD
}

This article was published in the following Dove Press journal:

Clinical Epidemiology

24 September 2012

Number of times this article has been viewed

\section{Pascale Salameh' \\ Georges Khayat ${ }^{2}$ \\ Mirna Waked ${ }^{3}$}

'Faculties of Pharmacy and of Public Health, Lebanese University, Beirut,

${ }^{2}$ Faculty of Medicine, Hôtel Dieu de France Hospital, Beirut and Saint Joseph University, Beirut, ${ }^{3}$ Faculty of Medicine, Saint George Hospital, Beirut and Balamand University, Beirut, Lebanon
Correspondence: Pascale Salameh Jdeidet El Meten, Chalet Suisse Street, Ramza Azzam Building, Fifth Floor,

Beirut, Lebanon 00961

Tel +96 I3385542

Fax +96 II696600

Email psalameh@ul.edu.lb
Background: Diagnosing chronic obstructive pulmonary disease (COPD) without spirometry is still a challenge. Our objective in this study was to develop a scale for diagnosis of COPD.

Methods: Data were taken from a cross-sectional epidemiological study. After reducing chronic respiratory symptoms, a logistic regression was used to select risk factors for and symptoms of COPD. The rounded coefficients generated a Diagnosis Score for COPD (DS-COPD), which was dichotomized and differentiated between COPD and other individuals with respiratory symptoms.

Results: We constructed a tool for COPD diagnosis with good properties, comprising 12 items. The area under the curve was 0.849 ; the positive predictive value was $76 \%$ if the DS-COPD was $>20$ and the negative predictive value was $97 \%$ if the DS-COPD was $<10$. A DS-COPD of $10-19$ represented a zone mostly suggestive of no COPD (77\%). The score was also inversely correlated with forced expiratory volume in 1 second/forced vital capacity.

Conclusion: In this study, a tool for diagnosis of COPD was constructed with good properties for use in the epidemiological setting, mainly in cases of low or high scoring. It would be of particular interest in the primary care setting, where spirometry may not be available. Prospective studies and application in clinical settings would be necessary to validate this scale further.

Keywords: diagnosis, scale, development, spirometry

\section{Introduction}

Chronic obstructive pulmonary disease (COPD) is a respiratory disease expected to rank third in 2020 in terms of global burden of disease. ${ }^{1}$ Due to the irreversible nature of COPD, early identification of the disease and its subsequent treatment is important. However, underdiagnosis is a worldwide problem. ${ }^{2}$

COPD is a genetically and environmentally complex disease, mainly related to smoking; however, some cases can also be caused by occupational exposure to toxic substances. ${ }^{2,3}$ Signs and symptoms differ among patients, leading to difficulties in diagnosis. ${ }^{3}$ Moreover, during the initial stages, COPD symptoms can be confused by patients and health professionals with aging, deconditioning, or other chronic conditions. ${ }^{4}$ The differential diagnosis with other respiratory diseases, such as asthma, further complicates the issue.

According to international guidelines, diagnosing COPD requires spirometry, a procedure mainly available in tertiary care hospitals. ${ }^{5,6}$ Although some portable spirometry devices are available, their use still requires considerable technical skill and patient learning effort. Further, widespread spirometric testing for early detection without 
preselection of at-risk patients may result in wasting of health care resources, ${ }^{7}$ which is of particular importance in primary care settings.

For these reasons, several authors have tried to predict COPD based on scoring systems, taking symptoms into account. Some have partially succeeded in coming up with new tools, but these have been of limited predictive value for COPD. ${ }^{8-13}$ Others have failed to obtain external validity of their scores. ${ }^{14}$ Thus, a tool able to predict COPD without spirometry has yet to be found, and our objective was to develop such a tool.

\section{Materials and methods Study design and population}

A cross-sectional study was carried out between October 2009 and September 2010 using a multistage cluster sample $(n=2201)$ across Lebanon. Lebanese residents aged 40 years and older were enrolled in the study, with no exclusion criteria. The total of males in this specific population was 614,564 , while the total of females was $653,751 .^{15}$ The institutional review board of the Lebanese University stated that ethics approval was not necessary in this case, given that the study was an observational one (neither interventional nor clinical). No approval number was allocated for this statement.

\section{Procedure}

From the list of communities in Lebanon (includes a total of 2782 villages, towns, and cities), ${ }^{16} 100$ communities were selected, with randomization performed using computer software. Individuals aged 40 years and above were then randomly chosen for an interview from a list of households provided via a representative of local authorities. All individuals in the household were solicited if they were eligible. After verbal informed consent was obtained, subjects underwent baseline spirometry (Micro Lab, Micro Medical Limited, Basingstoke, UK) conducted by a trained technician and answered a standardized questionnaire. Thirty minutes after inhalation of two puffs of ipratropium bromide (18 $\mu \mathrm{g}$ per actuation) and albuterol sulfate (Combivent ${ }^{\circledR}$, Boehringer Ingleheim, Germany, $103 \mu \mathrm{g}$ per actuation) in a pressurized metered-dose aerosol unit, a post-bronchodilator spirometry was performed. The best of three attempts was recorded. COPD was defined and classified according to Global Initiative for Obstructive Lung Disease (GOLD) guidelines (forced expiratory volume in 1 second/forced vital capacity $\left[\mathrm{FEV}_{1} / \mathrm{FVC}\right]<0.7$ after bronchodilation), and chronic bronchitis was defined as morning cough and sputum production for more than 3 months a year, for at least 2 years. ${ }^{6}$ Spirometric quality was checked, and $\mathrm{FEV}_{6} /$ FVC was $\leq 100 \%$ on more than $99.2 \%$ of measurements.

\section{Development of questionnaire}

The American Thoracic Society questionnaire for evaluation of chronic pulmonary diseases was used, ${ }^{17}$ which includes questions about all respiratory symptoms that are features of COPD, including chronic cough, sputum production, and wheezing. ${ }^{6}$ The Medical Research Council (MRC) scale was also used to evaluate dyspnea. ${ }^{18}$ In addition, the choice of symptoms was thoroughly discussed and subsequently approved by the two expert pulmonologists on the team, both of whom had had clinical experience for more than 15 years, to make sure that the list of symptoms was exhaustive. This list constituted the initial pool of items to be treated and adequately reduced.

The questionnaires were administered in the local Arabic language, translated as follows. First, two of the researchers, both bilingual, forward-translated the questions into Arabic, with instructions to translate conceptually rather than literally and to use familiar and easy language to target the broadest possible audience. Second, discrepancies were resolved by consensus between those researchers and two others, ie, the original translators and health experts, as well as experts with experience in instrument development and translation. Third, an independent translator with no knowledge of the questionnaire back-translated the questions into English. Translation discrepancies were resolved by consensus between the researchers and the translator. Fourth, the questionnaire was pilot-tested on 20 individuals; all questions were deemed clear by these individuals, and no further changes were made to the initial questions.

In addition to health questions, data concerning sociodemographic characteristics (eg, age, gender, education, marital status, dwelling region), and cigarette and waterpipe smoking history (current, previous, or never smokers) were collected. Cigarette smoking was defined as smoking more than one pack in a lifetime, while waterpipe smoking was defined as a cumulative dose of more than 15 waterpipeyears: this cumulative dose was calculated by multiplying the number of waterpipes per week by the duration of smoking in years. Information about weight, height, and any diagnosed cardiac problems was also gathered. Details on any additional environmental and occupational toxic exposure were also evaluated, such as exposure to smokes and fumes at work and at home, and any history of residence in polluted areas. 


\section{Calculation of sample size}

A minimal simple random sample size of $n=1015$ was required to measure the prevalence of COPD in an adult population aged 40 years and over in Lebanon, knowing that other studies have shown variation in COPD prevalence in the range of $9 \%-12 \%,{ }^{19,20}$ and taking a least acceptable result of $\pm 2 \%$ difference with the abovementioned prevalence and a $95 \%$ confidence interval (CI). To take into account the multistage sampling design further, a minimum sample size of 2030 individuals was necessary.

\section{Analysis}

SPSS version 17.0 (IBM, Armonk, NY) was used to enter and analyze the data. Weighting was performed according to figures published by the Lebanese Ministry of Social Affairs and Central Administration of Statistics in 2007, accounting for gender, age, and dwelling region, ${ }^{15}$ to improve sample representativeness. Cluster effect was also accounted for, according to Rumeau-Rouquette et al. ${ }^{21} P<0.05$ was considered to be statistically significant. The Chi-square test was used for cross-tabulation of qualitative variables, and concordance kappa values were calculated. Analysis of variance was used to compare means of quantitative variables, and Somers' D test was used to evaluate trends of ordinal variables. ${ }^{21}$

Next, an index was constructed using the initial list of COPD symptoms, and of all symptoms listed in the questionnaire, those that concorded best with a diagnosis of COPD were selected, ie, those with a kappa $>0.1$. In this way, the COPD Symptoms Index was generated. The construct validity of this index was evaluated by factorial analysis, whereby the index items were plotted in a factorial analysis to reduce their number, and after ensuring adequate Kaiser-Meyer-Olkin and sample adequacy, promax rotations were used and factor loading was recorded. A reliability analysis was also performed and Cronbach's alpha was calculated, along with sensitivity, specificity, and negative and positive predictive values.

A logistic regression was then run with COPD as a dependent variable, while the independent variables included sociodemographic characteristics (age, class, gender, and education), risk factors for COPD (previous and actual cigarette and waterpipe smoking, cooking on wood, heating on diesel, heating on gas, heating on wood, passive smoking, living close to a road with heavy traffic, occupational exposure to gases and fumes, and living close to electricity generators), and the reduced list of symptoms (cough throughout the day, sputum production throughout the day, MRC dyspnea score, morning sputum production, morning cough, and wheezing throughout the day). The final model was subjected to Hosmer and Lemeshow testing for adequacy of the data; collinearity was also tested to ensure its absence between dependent variables. The adjusted odds ratios (OR) obtained were then rounded to the nearest units and used as coefficients in the subsequent score, ie, the Diagnosis Score for COPD (DS-COPD). This calculated score was applied for validation, ie, receiver-operating characteristic curves were used to determine a threshold for the index with best sensitivity, specificity, and positive and negative predictive values. Subgroup analyses of symptomatic individuals were also performed.

\section{Results}

\section{Summary of primary results}

The primary results of the study were analyzed, and only $33.3 \%$ of 2201 individuals had never smoked. The prevalence of COPD by GOLD definition was $9.7 \%$ (95\% CI 8.5-10.9). Among them, 20.2\% were already diagnosed by a physician. No differences in symptoms across the stages of COPD were found, but there was a significant trend towards a higher number of visits to the emergency room and to the doctor $(P<0.001)$ and a higher number of hospitalizations $(P<0.001)$. Older individuals had an increased risk of COPD (adjusted OR 1.05) and being ever cigarette smokers (OR 4.88) or waterpipe smokers (OR 2.53). Further details and results of this study have been published elsewhere. ${ }^{22}$

\section{Reduction in chronic symptoms reported}

Table 1 shows the prevalence of spirometrically defined COPD in the presence or absence of respiratory symptoms, along with the kappa concordance coefficient for every symptom. It was found that COPD was best predicted (kappa coefficient $>0.1$ ) by chronic cough, morning cough, chronic sputum production, morning sputum production, all day sputum, any wheezing, all day wheezing, and if cold weather triggers a lung problem. Moreover, a higher dyspnea score was associated with an increase in the prevalence of COPD $(P<0.001$ for trends)

\section{Construct validity and reliability of COPD Symptoms Index}

Five symptoms gave a Kappa concordance coefficient value above 0.1 , thus allowing construction of the COPD Symptoms Index. This index included morning cough, 
Table I Reported chronic symptoms, spirometry, and COPD

\begin{tabular}{|c|c|c|c|c|c|}
\hline Symptoms & Number (total) & Normal spirometry & $P$ value & COPD (\%) & $\begin{array}{l}\text { Kappa; } \\
\text { P value }\end{array}$ \\
\hline Chronic cough & & & $<0.001$ & & 0.252 \\
\hline No & 1690 & $77.7 \%$ & & $7.9 \%$ & $<0.001$ \\
\hline Yes & 511 & $45.4 \%$ & & $29.2 \%$ & \\
\hline Morning cough & & & $<0.001$ & & 0.117 \\
\hline No & 1973 & $71.9 \%$ & & $11.6 \%$ & $<0.00$ I \\
\hline Yes & 228 & $55.1 \%$ & & $24.6 \%$ & \\
\hline Evening cough & & & 0.009 & & 0.037 \\
\hline No & 2161 & $70.5 \%$ & & $12.6 \%$ & 0.005 \\
\hline Yes & 40 & $51.3 \%$ & & $27.5 \%$ & \\
\hline Night cough & & & $<0.001$ & & $0.05 \mathrm{I}$ \\
\hline No & 2100 & $71.3 \%$ & & $12.4 \%$ & 0.005 \\
\hline Yes & 100 & $47.0 \%$ & & $22.0 \%$ & \\
\hline Cough all day & & & $<0.001$ & & 0.301 \\
\hline No & 2005 & $74.5 \%$ & & $9.7 \%$ & $<0.001$ \\
\hline Yes & 196 & $26.0 \%$ & & $45.9 \%$ & \\
\hline Chronic sputum & & & $<0.001$ & & $0.27 I$ \\
\hline No & 1632 & $77.8 \%$ & & $7.0 \%$ & $<0.00$ I \\
\hline Yes & 569 & $48.3 \%$ & & $29.7 \%$ & \\
\hline Morning sputum & & & $<0.001$ & & 0.146 \\
\hline No & 1905 & $72.8 \%$ & & $11.0 \%$ & $<0.001$ \\
\hline Yes & 296 & $53.7 \%$ & & $25.3 \%$ & \\
\hline Evening sputum & & & 0.043 & & 0.033 \\
\hline No & 2163 & $70.4 \%$ & & $12.7 \%$ & 0.024 \\
\hline Yes & 38 & $55.3 \%$ & & $26.3 \%$ & \\
\hline Night sputum & & & $<0.001$ & & 0.043 \\
\hline No & 2132 & $71.1 \%$ & & $12.5 \%$ & 0.008 \\
\hline Yes & 68 & $42.6 \%$ & & $23.5 \%$ & \\
\hline Sputum all day & & & $<0.001$ & & 0.273 \\
\hline No & 2012 & $73.6 \%$ & & $10.0 \%$ & $<0.00$ I \\
\hline Yes & 188 & $34.0 \%$ & & $43.6 \%$ & \\
\hline Any wheezing & & & $<0.001$ & & $0.24 I$ \\
\hline No & 1575 & $78.3 \%$ & & $7.2 \%$ & $<0.001$ \\
\hline Yes & 626 & $49.8 \%$ & & $27.3 \%$ & \\
\hline Morning wheezing & & & $<0.001$ & & 0.039 \\
\hline No & 2085 & $71.2 \%$ & & $12.6 \%$ & 0.041 \\
\hline Yes & 115 & $51.7 \%$ & & $19.1 \%$ & \\
\hline Evening wheezing & & & $<0.001$ & & 0.059 \\
\hline No & 2131 & $70.5 \%$ & & $12.4 \%$ & $<0.001$ \\
\hline Yes & 70 & $58.6 \%$ & & $27.1 \%$ & \\
\hline Night wheezing & & & 0.031 & & 0.074 \\
\hline No & 2000 & $71.9 \%$ & & $12.1 \%$ & $<0.001$ \\
\hline Yes & 201 & $52.7 \%$ & & $20.9 \%$ & \\
\hline Wheezing all day & & & $<0.001$ & & 0.213 \\
\hline No & 2048 & $72.9 \%$ & & $10.8 \%$ & $<0.001$ \\
\hline Yes & 153 & $33.3 \%$ & & $40.5 \%$ & \\
\hline Cold becomes a lung problem & & & $<0.001$ & & 0.211 \\
\hline No & 1712 & $76.3 \%$ & & $8.9 \%$ & $<0.001$ \\
\hline Yes & 488 & $48.6 \%$ & & $26.8 \%$ & \\
\hline Any chronic allergy & & & 0.339 & & 0.017 \\
\hline No & 1722 & $70.7 \%$ & & $12.5 \%$ & 0.395 \\
\hline Yes & 478 & $68.4 \%$ & & $14.0 \%$ & \\
\hline Allergic rhinitis & & & 0.885 & & 0.004 \\
\hline No & 1906 & $70.1 \%$ & & $12.9 \%$ & 0.861 \\
\hline Yes & 295 & $70.5 \%$ & & $13.2 \%$ & \\
\hline
\end{tabular}


Table I (Continued)

\begin{tabular}{|c|c|c|c|c|c|}
\hline Symptoms & Number (total) & Normal spirometry & $P$ value & COPD (\%) & $\begin{array}{l}\text { Kappa; } \\
\text { P value }\end{array}$ \\
\hline Lung allergy & & & $<0.001$ & & 0.068 \\
\hline No & 2094 & $71.2 \%$ & & $12.3 \%$ & $<0.001$ \\
\hline Yes & 106 & $50.5 \%$ & & $24.5 \%$ & \\
\hline At least one symptom & & & $<0.001$ & & 0.200 \\
\hline Yes & 1112 & $54.1 \%$ & & $22.7 \%$ & $<0.001$ \\
\hline No & 1088 & $86.5 \%$ & & $2.8 \%$ & \\
\hline MRC dyspnea scale & Number (total) & Normal spirometry & $P$ value & COPD* & OR/kappa \\
\hline 0 & 1267 & $80.2 \%$ & Reference & $7.1 \%$ & 1.00 \\
\hline I & 325 & $64.6 \%$ & $<0.001$ & $12.0 \%$ & 1.78 \\
\hline 2 & 144 & $66.7 \%$ & $<0.001$ & $17.2 \%$ & 2.72 \\
\hline 3 & 149 & $58.4 \%$ & $<0.001$ & $10.8 \%$ & 1.58 \\
\hline 4 & 146 & $50.7 \%$ & $<0.00$ I & $26.0 \%$ & 4.60 \\
\hline 5 & 170 & $35.7 \%$ & $<0.00$ I & $44.7 \%$ & 10.56 \\
\hline MRC score $>0$ & & & $<0.00 \mathrm{I}$ & & \\
\hline No & 1267 & $80.2 \%$ & & $7.1 \%$ & 0.150 \\
\hline Yes & 934 & $56.5 \%$ & & $20.8 \%$ & \\
\hline
\end{tabular}

Abbreviations: COPD, chronic obstructive pulmonary disease; MRC, Medical Research Council; OR, odds ratio.

morning sputum, all day sputum, all day wheezing, and MRC dyspnea score. In the factorial analysis, three factors were retained, explaining $78 \%$ of the total variance: three items loaded on one factor (all day cough, all day sputum production, and MRC dyspnea score), two other items loaded on a second factor ("chronic bronchitis" factor, including morning cough and sputum production), while the last item loaded on a third factor (all day wheezing). This index had good reliability (alpha 0.692). However, the sensitivity of this score (if higher than zero) was $82.9 \%$ and its specificity was $56.6 \%$. The positive predictive value was $16.8 \%$ and the negative predictive value was $96.9 \%$; these values were deemed to be of low interest (Table 2).

\section{Construction of DS-COPD}

The logistic regression for factors predicting COPD in this cross-sectional study is presented in Table 3. The model finally retained suggested the following: older age, male gender, and low birth weight are nonmodifiable risk factors for COPD; home heating with diesel, cooking on wood, previous or current cigarette smoking, and previous waterpipe smoking are factors associated with COPD. Associated symptoms include higher MRC score, all day coughing, all day wheezing, and chronic bronchitis.

Taking into account the adjusted OR and rounding to the nearest unit, the DS-COPD was computed as follows: DS-COPD $=($ previous waterpipe smoking*3 $)+($ MRC score $)+($ age class $)+($ gender $)+$ (heating home by diesel*2) + (cooking on wood*3) + (low birth weight $)+($ current cigarette smoking*3) $+($ previous cigarette smoking*3) $+($ all day coughing*2) + (chronic bronchitis*2) + (all day wheezing*2).

The DS-COPD has a minimum of two and a maximum of 33 points. In the sample, the minimum was two and the maximum was 27. In individuals with COPD, the mean was 14.98 , the median 15 , and the standard deviation 4.64 , while in healthy controls, the mean was 6.09 , the median 6 , and the standard deviation $2.96(P<0.001)$.

Table 2 Construct validity and reliability of COPD Symptoms Index (scale*)

\begin{tabular}{llll}
\hline Scale items & Loading on factor I & Loading on factor 2 & Loading on factor $\mathbf{3}$ \\
\hline Cough all day & 0.909 & & \\
Sputum production all day & 0.898 & \\
MRC score & 0.420 & 0.888 & \\
Morning sputum production & & 0.864 & \\
Morning cough & & & 1.006 \\
Wheezing all day & &
\end{tabular}

Note: *Reliability Cronbach's alpha $=0.692$.

Abbreviations: COPD, chronic obstructive pulmonary disease; MRC, Medical Research Council. 
Table 3 Logistic regression* for COPD correlates

\begin{tabular}{|c|c|c|c|}
\hline Factor & aOR & $95 \% \mathrm{Cl}$ & $P$ value \\
\hline Age class & 1.49 & $1.31-1.70$ & $<0.001$ \\
\hline Female gender & 0.55 & $0.38-0.80$ & 0.002 \\
\hline Low birth weight & 1.27 & $1.00-1.61$ & 0.053 \\
\hline Heating home by diesel & 1.49 & $\mathrm{I} .0 \mathrm{I}-2.20$ & 0.046 \\
\hline Cooking on wood & 2.75 & I.39-5.46 & 0.004 \\
\hline Actual cigarette smoking & 3.33 & $2.02-5.49$ & $<0.001$ \\
\hline Previous cigarette smoking & 2.87 & I.75-4.72 & $<0.001$ \\
\hline Previous waterpipe smoking & 2.60 & I.32-5.13 & 0.006 \\
\hline MRC score & 1.24 & $1.12-1.38$ & $<0.00$ I \\
\hline Coughing all day & 2.18 & $1.34-3.56$ & 0.002 \\
\hline Chronic bronchitis & 1.62 & $1.10-2.39$ & 0.015 \\
\hline Wheezing all day & 1.61 & $0.96-2.70$ & 0.072 \\
\hline
\end{tabular}

Notes: $*$ Nagelkerke $\mathrm{R}$ square $=0.339$; Hosmer and Lemeshow adequacy test $P=0.406 ; 91.1 \%$ of individuals were correctly classified; dependent variable is COPD, while the independent variables include sociodemographic characteristics (age class, gender, and education), risk factors for COPD (previous and actual cigarette and waterpipe smoking, cooking on wood, heating on diesel, heating on gas, heating on wood, passive smoking, living close to a road with heavy traffic, occupational exposure to gases and fumes, living close to electricity generators), and symptoms (cough all day, sputum production all day, MRC dyspnea score, morning sputum production, morning cough, and wheezing all day).

Abbreviations: aOR, adjusted odds ratio; MRC, Medical Research Council; $\mathrm{Cl}$, confidence interval; COPD, chronic obstructive pulmonary disease.

\section{DS-COPD properties and thresholds}

Receiver-operating characteristic curves for COPD prediction are shown in Figure 1, comparing COPD patients with all others. The area under the curve was high at 0.849 (0.826-0.873; $P<0.001)$. However, a unique threshold with both good sensitivity and specificity was difficult to

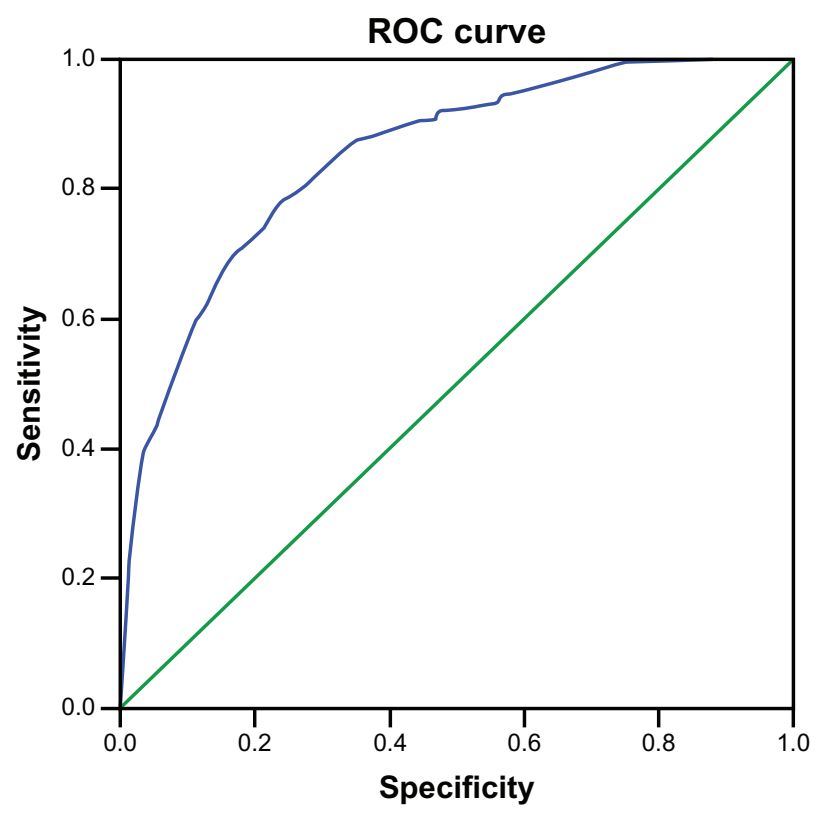

Figure I Receiver-operating characteristic curve for threshold selection of Diagnosis Score for Chronic Obstructive Pulmonary Disease score.

Note: Area under the curve $=0.849(0.826-0.873, P<0.001)$.

Abbreviation: ROC, receiver-operating characteristic curve. identify (Table 4). For example, on threshold 9, a sensitivity of 0.91 was found but with a specificity of 0.53 , while on threshold 17 , a specificity of 0.97 was found but with a sensitivity of 0.40 .

\section{DS-COPD and prediction of COPD}

When recoding the DS-COPD into five-point categories, increased predictive values for COPD were obtained with increased DS-COPD (Figure 2). Extreme cases were a score $\leq 4$ with a negative predictive value of $100 \%$, while a score $\geq 25$ had a positive predictive value of $88.9 \%$. Very similar results were found for symptomatic individuals. Based on these figures, two thresholds appear to be the most appropriate, ie, a DS-COPD $\leq 9$ points has an excellent predictive value for no COPD $(96.5 \%$ no COPD versus $3.5 \%$ COPD), while a DS-COPD of $\geq 20$ points has a good predictive value for COPD ( $76 \%$ COPD versus $24 \%$ no COPD).

\section{DS-COPD and disease severity}

The regression equation was calculated after ensuring relationship linearity between DS-COPD score and post-bronchodilator $\mathrm{FEV}_{1} / \mathrm{FVC}$. The following equation was obtained:

$$
\left[\mathrm{FEV}_{1} / \mathrm{FVC}\right]=83.48-(0.8 *[\mathrm{DS}-\mathrm{COPD}])
$$

Moreover, based on the thresholds chosen above, significant differences in the number of emergency visits in the previous year for respiratory problems were noticed, ie, 0.02 if the DS-COPD score was $0-9$ versus 0.28 if the DS-COPD score was $1-19$ versus 0.48 if the DS-COPD score was $\geq 20(P<0.001)$. An analogous trend was found for the number of hospitalizations of more than 1 day due to respiratory problems, ie, 0.02 versus 0.24 versus 0.38 , respectively $(P<0.001)$, and for the number of visits to the doctor because of respiratory problems $(0.07$ versus 0.45 versus 0.79 respectively; $P<0.001$ ).

\section{Discussion}

In this study, a tool with good properties was constructed for diagnosis of COPD, comprising 12 sociodemographic characteristics, previous and present toxic exposure and smoking history, in addition to chronic respiratory symptoms. These scale properties are better than those devised by other researchers. ${ }^{8-13}$ Although we did not focus on high-risk populations, such as smokers, patients previously diagnosed with respiratory diseases, ${ }^{8,9}$ individuals consulting for respiratory problems, ${ }^{11}$ or those with reported chronic bronchitis, ${ }^{13}$ like in other studies, we did obtain a good area under the curve 
Table 4 Coordinates of the receiver-operating characteristics curve

\begin{tabular}{|c|c|c|}
\hline COPD positive if $\geq(a)$ & Sensitivity & Specificity \\
\hline 1.0000 & 1.000 & 1.000 \\
\hline 2.1667 & 1.000 & 0.997 \\
\hline 2.6667 & 1.000 & 0.975 \\
\hline 3.1667 & 1.000 & 0.970 \\
\hline 3.6667 & 1.000 & 0.888 \\
\hline 4.1667 & 1.000 & 0.884 \\
\hline 4.3333 & 1.000 & 0.884 \\
\hline 4.6667 & 0.997 & 0.828 \\
\hline 5.1667 & 0.997 & 0.824 \\
\hline 5.3333 & 0.997 & 0.824 \\
\hline 5.6667 & 0.993 & 0.753 \\
\hline 6.1667 & 0.993 & 0.746 \\
\hline 6.3333 & 0.993 & 0.746 \\
\hline 6.6667 & 0.969 & 0.661 \\
\hline 7.1667 & 0.965 & 0.656 \\
\hline 7.3333 & 0.965 & 0.656 \\
\hline 7.6667 & 0.944 & 0.568 \\
\hline 8.1667 & 0.934 & 0.562 \\
\hline 8.3333 & 0.927 & 0.523 \\
\hline 8.6667 & 0.920 & 0.473 \\
\hline 9.1667 & 0.906 & 0.467 \\
\hline 9.3333 & 0.906 & 0.449 \\
\hline 9.6667 & 0.885 & 0.380 \\
\hline 10.1667 & 0.882 & 0.376 \\
\hline 10.3333 & 0.875 & 0.350 \\
\hline 10.6667 & 0.812 & 0.280 \\
\hline II.1667 & 0.808 & 0.278 \\
\hline 11.3333 & 0.780 & 0.238 \\
\hline II.6667 & 0.742 & 0.214 \\
\hline 12.1667 & 0.735 & 0.212 \\
\hline 12.3333 & 0.711 & 0.182 \\
\hline 12.6667 & 0.690 & 0.164 \\
\hline 13.1667 & 0.690 & 0.161 \\
\hline 13.3333 & 0.606 & 0.122 \\
\hline 13.6667 & 0.599 & 0.114 \\
\hline 14.1667 & 0.596 & 0.112 \\
\hline 14.3333 & 0.537 & 0.091 \\
\hline |4.6667 & 0.516 & 0.083 \\
\hline 15.1667 & 0.512 & 0.082 \\
\hline 15.3333 & 0.446 & 0.058 \\
\hline 15.6667 & 0.443 & 0.057 \\
\hline 16.1667 & 0.439 & 0.057 \\
\hline 16.6667 & 0.397 & 0.037 \\
\hline 17.1667 & 0.397 & 0.036 \\
\hline 17.8333 & 0.314 & 0.022 \\
\hline 18.6667 & 0.254 & 0.016 \\
\hline 19.1667 & 0.251 & 0.016 \\
\hline 19.8333 & 0.195 & 0.012 \\
\hline 20.8333 & 0.122 & 0.010 \\
\hline 21.6667 & 0.087 & 0.006 \\
\hline 22.1667 & 0.080 & 0.006 \\
\hline 22.8333 & 0.049 & 0.003 \\
\hline 23.8333 & 0.017 & 0.002 \\
\hline 24.8333 & 0.014 & 0.001 \\
\hline 25.8333 & 0.007 & 0.000 \\
\hline 26.8333 & 0.003 & 0.000 \\
\hline 28.3333 & 0.000 & 0.000 \\
\hline
\end{tabular}

value for our whole sample. These good results may be due to the fact that different types of risk factors (modifiable and nonmodifiable) were taken into account, in addition to chronic respiratory symptoms. Indeed, taking symptoms alone gave a scale with a poor ability to predict COPD, and this was similar to the results of Hill et al. ${ }^{23}$

Thus, by adding risk factors, a higher percentage of disease variability can be explained, and the DS-COPD may be a conceptual and clinical advance over prior assessment scores. In addition to age, low birth weight and gender are known to be risk factors for COPD. ${ }^{25}$ Five behavioral/environmental risk factors were also involved in COPD, ie, previous and current cigarette smoking, and cooking on wood and heating home by diesel. These factors have been associated with COPD. ${ }^{24-27}$ Previous waterpipe smoking of more than 15 weekly waterpipes-years also seems to be a risk factor for COPD; another specificity of this scale, the association between waterpipe smoking and COPD has been found by ourselves and others. ${ }^{27}$

Moreover, chronic respiratory symptoms were included in the scale (chronic bronchitis, MRC dyspnea, all day coughing, and wheezing), and although other researchers took presence and frequency of symptoms into consideration, ${ }^{8-13}$ this is one of the rare scales that includes timing of symptoms, such as morning sputum production and cough for chronic bronchitis and all day wheezing and coughing. An exception to this was found for Price et al, who took morning sputum production into account, but with no timing reported for other symptoms. ${ }^{9}$ In fact, COPD symptoms do have a circadian rhythm, with morning symptoms being mostly reported by patients, ${ }^{28}$ while for other chronic respiratory diseases such as asthma, symptoms are known to worsen during the night and early morning hours. ${ }^{29}$

Our scale also had a good ability to differentiate between COPD and non-COPD patients with respiratory problems, ie, in individuals with chronic respiratory symptoms, still better than all other previously reported scales. ${ }^{8-13}$ Thus, the scale constructed here can be used in settings where spirometry is not available. It has best predictive ability for COPD in symptomatic patients, but has no utility in asymptomatic individuals (in which case the COPD prevalence is $0.7 \%$ and the positive predictive value is $0.9 \%$, results not shown). Moreover, it was demonstrated here that the DS-COPD score is inversely correlated with COPD severity. Overall, a score $\geq 20$ is suggestive of COPD (76\%), a score $\leq 9$ applies mainly to individuals with no COPD $(93 \%$, low predictive value for COPD). A score of 10-19 seems to be a zone of miscellaneous symptomatic respiratory disease, most probably 


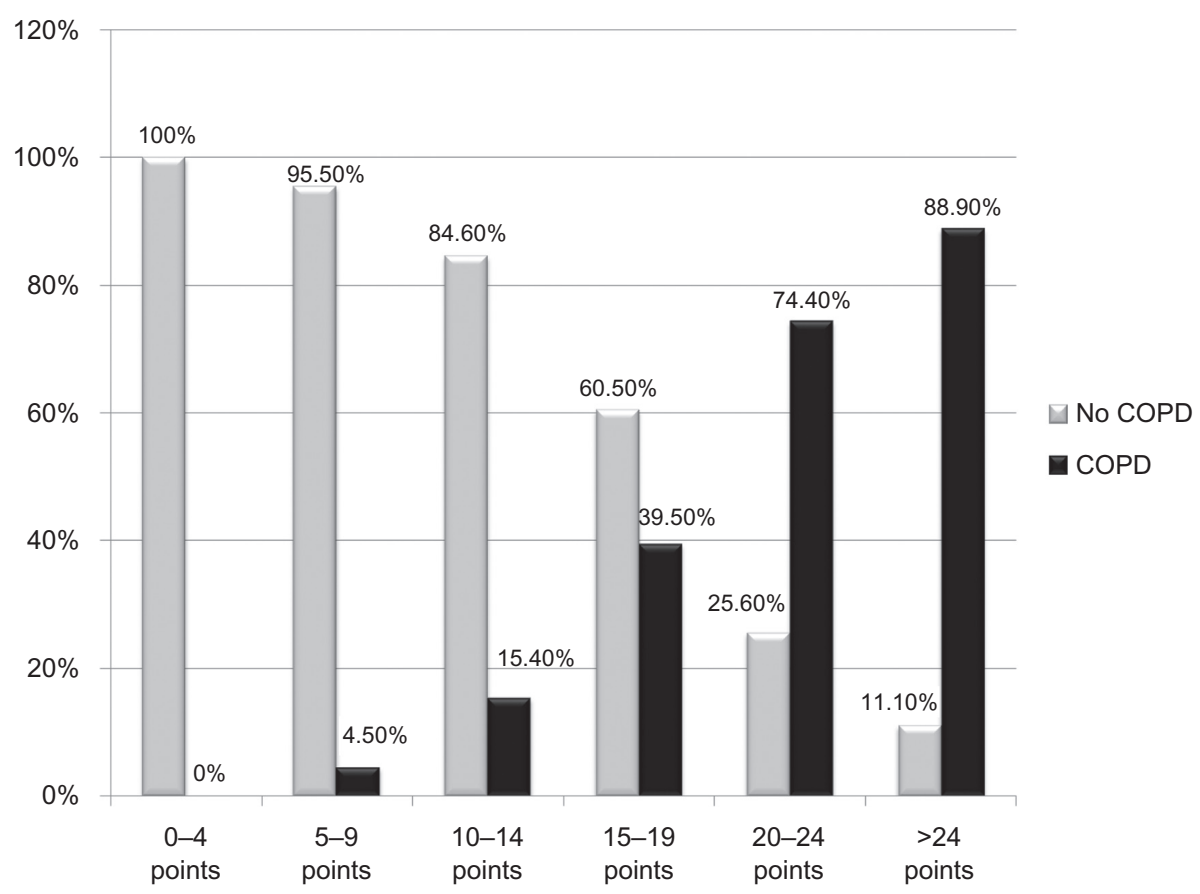

Figure 2 Percentages of COPD and of respiratory symptoms (not COPD) by category of Diagnosis Score for COPD in symptomatic individuals. Abbreviation: COPD, chronic obstructive pulmonary disease.

suggestive of no COPD (77\%), and in the latter case, the absence of COPD could only be confirmed by spirometry.

This new scale should be tested in prospective studies to check its value in clinical settings, its test-retest reliability, and its responsiveness to changes in patient COPD status. Some associations, such as occupational exposure to toxics and fumes and environmental exposure to toxics, such as living near roads with heavy traffic, did not reach statistical significance, but this could be due to sample size. Other limitations of the present work include the possibility of selection bias and information bias coupled with the questionnaire used. Cooking with oil is also a risk factor for COPD and was not taken into account.

The demonstrated dose-effect relationship and multivariate analysis are considered to be the strong points of this work. However, given that these data were collected from a Lebanese population, and factors related to the Mediterranean region, such as cooking on wood stoves and waterpipe smoking, may not be generalizable to the global COPD population, crosscultural validation is suggested. Additional studies should take into account phenotypic variations in COPD (ie, the chronic bronchitis and emphysema subtypes), linking them to genes that could be specific to the Lebanese population.

In conclusion, we were able to construct a tool for COPD diagnosis with good properties in an epidemiological setting, mainly for symptomatic individuals, that correlated inversely with disease severity. This tool would be particularly useful for management of patients with COPD before availability of spirometry results or in the absence of spirometry. Its value remains to be confirmed in future prospective clinical studies.

\section{Acknowledgment}

This study is part of a project funded by an educational grant from the Lebanese University.

\section{Disclosure}

Researchers have no conflict of interest to declare in this work.

\section{References}

1. Murray CJ, Lopez AD. Alternative projections of mortality and disability by cause 1990-2020: Global Burden of Disease Study. Lancet. 1997;349:1498-1504.

2. Pauwels PRA, Rabe KF. Burden and clinical features of chronic obstructive pulmonary disease (COPD). Lancet. 2004;364:613-620.

3. Molfino N. Genetics of COPD. Chest. 2004;125:1929-1940.

4. Radin A, Cote C. Primary care of the patient with chronic obstructive pulmonary disease - Part 1: frontline prevention and early diagnosis. Am J Med. 2008;121(Suppl 7):S3-S12.

5. Celli BR, MacNee W; ATS/ERS Task Force. Standards for the diagnosis and treatment of patients with COPD: a summary of the ATS/ERS position paper. Eur Respir J. 2004;23:932-946.

6. Rabe KF, Hurd S, Anzueto A, et al; Global Initiative for Chronic Obstructive Lung Disease. Global strategy for the diagnosis, management, and prevention of chronic obstructive pulmonary disease: GOLD executive summary. Am J Respir Crit Care Med. 2007;176:532-555. 
7. Enright P. Does screening for COPD by primary care physicians have the potential to cause more harm than good? Chest. 2006;129:833-835.

8. Price DB, Tinkelman DG, Halbert RJ, et al. Symptom-based questionnaire for identifying chronic obstructive pulmonary disease in smokers. Respiration. 2006;73:285-295.

9. Price DB, Tinkelman DG, Nordyke RJ, Isonaka S, Halbert RJ; COPD Questionnaire Study Group. Scoring system and clinical application of COPD diagnostic questionnaires. Chest. 2006;129:1531-1539.

10. Kida K, Wakabayashi R, Mizuuchi T, Murata A. Screening for suspected chronic obstructive pulmonary disease with an eleven-item pre-interview questionnaire (11-Q). Intern Med. 2006;45:1201-1207.

11. Martinez F, Raczek A, Seifer FD, et al. Development and initial validation of a self-scored COPD population screener questionnaire (COPD-PS). COPD. 2008;5:85-95.

12. Bailey WC, Sciurba FC, Hanania NA, et al. Development and validation of the chronic obstructive pulmonary disease assessment questionnaire (COPD-AQ). Prim Care Respir J. 2009;18:198-207.

13. Yawn BP, Mapel DW, Mannino DM, et al. On behalf of the Lung Function Questionnaire Working Group. Development of the lung function questionnaire (LFQ) to identify airflow obstruction. COPD. 2010;5:1-10.

14. Kotz D, Nelemans P, van Schayck CP, Wesseling GJ. External validation of a COPD diagnostic questionnaire. Eur Respir J. 2008;31:298-303.

15. Lebanese Ministry of Social Affairs and Central Administration of Statistics. The National Study for Household Living Conditions in 2007. Beirut, 2008. Available from: http://www.cas.gov.lb. Accessed on May $15,2012$.

16. Central Administration of Statistics. Index of circumscriptions, villages and cities in Lebanon. Jun 2005, Beirut, Lebanon. Available from: http:// www.cas.gov.lb. Accessed on May 16, 2012.

17. Ferris BG. Epidemiology standardization project. Am Rev Respir Dis. 1978;118:1-88.

18. Fletcher CM, Elmes PC, Wood CH. The significance of respiratory symptoms and the diagnosis of chronic bronchitis in a working population. BMJ. 1959;2:257-266.
19. Soriano JB, Ancochea J, Miravitlles M, et al. Recent trends in COPD prevalence in Spain: a repeated cross-sectional survey 1997-2007. Eur Respir J. 2010;36:758-765.

20. Caballero A, Torres-Duque CA, Jaramillo C, et al. Prevalence of COPD in five Colombian cities situated at low, medium, and high altitude. Chest. 2008;133:343-349.

21. Rumeau-Rouquette C, Breart G, Padieu R. Methods in Epidemiology: Sampling, Investigations and Analysis. Paris, France: Flammarion; 1985.

22. Waked M, Khayat P, Salameh P. COPD prevalence in Lebanon: A cross-sectional descriptive study. Clin Epidemiol. 2011;3:315-323.

23. Hill K, Hodder R, Blouin M, Heels-Ansdell D, Guyatt G, Goldstein R. Identifying adults at risk of COPD who need confirmatory spirometry in primary care: do symptom-based questions help? Can Fam Physician 2011;57:e51-e57.

24. Fletcher CM, Peto R. The natural history of chronic airflow obstruction. BMJ. 1977;1:1645-1648.

25. Griffith KA, Sherrill DL, Siegel EM, Manolio TA, Bonekat HW, Enright PL. Predictors of loss of lung function in the elderly. The Cardiovascular Health Study. Am J Respir Crit Care Med. 2001;163: 61-68.

26. Zhang JJ, Smith KR. Household air pollution from coal and biomass fuels in China: measurements, health impacts, and interventions. Environ Health Perspect. 2007;115:848-855.

27. Maziak W, Rastam S, Ibrahim I, Ward KD, Shihadeh A, Eissenberg T. CO exposure, puff topography, and subjective effects in waterpipe tobacco smokers. Nicotine Tob Res. 2009;11:806-811.

28. Partridge MR, Karlsson N, Small IR. Patient insight into the impact of chronic obstructive pulmonary disease in the morning: an Internet survey. Curr Med Res Opin. 2009;25:2043-2048.

29. Burioka N, FukuokaY, Koyanagi S, et al. Asthma: chronopharmacotherapy and the molecular clock. Adv Drug Deliv Rev. 2010;62:946-955.
Clinical Epidemiology

\section{Publish your work in this journal}

Clinical Epidemiology is an international, peer-reviewed, open access journal focusing on disease and drug epidemiology, identification of risk factors and screening procedures to develop optimal preventative initiatives and programs. Specific topics include: diagnosis, prognosis, treatment, screening, prevention, risk factor modification, systematic

\section{Dovepress}

reviews, risk \& safety of medical interventions, epidemiology \& biostatical methods, evaluation of guidelines, translational medicine, health policies \& economic evaluations. The manuscript management system is completely online and includes a very quick and fair peer-review system, which is all easy to use. 